\title{
Prevention of Hepatitis in People Living With HIV
}

\author{
Bhuvaneshwari $\mathrm{S}^{1 *}$, Manickavalli $\mathrm{E}^{2}$, Apollo James ${ }^{3}$, Mercy \\ Dora. $T^{4}$ \\ ${ }^{1}$ Associate Professor, Department of Pharmaceutical Biotechnology, Nandha College of \\ Pharmacy, Erode, Tamilnadu, Email:s.sribhu@rediffmail.com \\ ${ }^{2}$ Assistant Professor, Department of Pharmaceutical Chemistry, Nandha College of \\ Pharmacy, Erode, Tamilnadu, Email:manickavallielangovan@gmail.com \\ ${ }^{3}$ Associate Professor, Department of Pharmacy Practice, Nandha College of Pharmacy, \\ Erode, Tamilnadu, Email: ajamespharma@gmail.com \\ ${ }^{4}$ Associate Professor,Mental Health Nursing, Nandha College of Nursing, Erode, \\ Tamilnadu, Email:mercymurali@gmail.com \\ ${ }^{*}$ Corresponding Author
}

\begin{abstract}
The dominance of hepatitis $\mathrm{C}$ virus (HCV) and hepatitis virus (HBV) infections among people infected with HIV is high and increasing, unlike the rest of the realm. Nevertheless, a small number of HIVinfected patients receive treatment for hepatitis. Altering the integrity of co-infection outcomes makes the design of such patients a major challenge. Efforts should also be thru through multidisciplinary wellbeing overhaul amenities to surge the relevance and accessibility of care, particularly for vulnerable groups such as migrants, injectable drug users (IDUs), inmates, and people with drug conditions. A person who consumes excessive amounts of alcohol. The persistence of this article is to deliver a clear idea of the treatment outcomes and treatment of our HIV infection with HAV, HBV and HCV.
\end{abstract}

Keywords: HCV, HBV, Injectable drug

\section{Introduction}

Hepatitis is a disease in which the liver becomes inflamed. Although usually the result of an infection in the body, the causes of infectious diseases such as hepatitis and hepatitis can vary due to secondary exposure to drugs, drugs, pollutants, and alcohol. Reactive hepatitis can be a ailment that befalls after the physique marks antibodies to liver tissue. There are five main categories of hepatitis pathogens: hepatitis A, B, C, D, and E. Each type of viral hepatitis causes a specific virus. The World Health Organization (WHO) currently estimates that 354 million individuals worldwide have chronic hepatitis B and C.

This form of hepatitis is a critical, stopgap disease. Serum hepatitis virus (HBV) causes hepatitis B. It is a chronic disease that usually lasts. Viral hepatitis is triggered by the hepatitis $\mathrm{C}$ virus (HCV). $\mathrm{HCV}$ is one of the most joint

Blood abided contaminations in the countries, occurs regularly and often grants as a long-standing illness. This may be a erratic form of hepatitis that merely ensues with hepatitis B septicity. Hepatitis D virus (HDV), like many strains, causes liver inflammation, but no one can become infected with HDV until infected with serum of hepatitis B. Disease E Transmitted through water contact with the hepatitis $E$ virus (HEV). Liver disease $E$ is common in expanses with pitiable sanitation and is often caused by ingestion of waste products that contaminate water. People with AIDS become very weak and must be infected with many replacement bacteria. Human immunodeficiency virus (HIV) may be a virus that enters the bloodstream. In people who are HIVpositive, the illness can be regularly detected in blood, sexual fluids (such as semen and tubules), and breast milk.

HIV size is too trivial to be seen with the naked eye. HIV outbreaks and ultimately rescinds body systems. The system is like a defense force of the body. It always fights germs, infections, organisms and viruses that can cause disease. When this "army" weakens, the body cannot shield the aforementioned against germs, infections, germs, and viruses. HIV causes AIDS (Acquired Immunodeficiency Syndrome). HIV slowly indemnities the immune system when it enters the human body. This means that the body begins to lose its ability to shield itself against germs, infections, and bacteria. People with AIDS become much debilitated and have to become infected with many replacement bacteria.

\section{HIV Co-infection and Disease}

It is a common disease and contributes significantly to indisposition and transience in people living with HIV (PLHIV). The purpose of this study was to govern the 
pervasiveness of these co-infections in patients receiving second-line antiretroviral therapy. Coinfection with HBV and HCV is common in HIV-infected patients due to overlapping routes of transmission3. Over time, the introduction of high activity antiretroviral therapy (HAART) expressively condensed ill health and impermanence in HIVinfected patient. Therefore, the importance of comorbidities similar to chronic diseases caused by $\mathrm{HBV}$ and HCV infection is recognized as an important issue. HIV infection alters the cause of chronic parenteral nontransferable viral hepatitis with very rapid disease progression. Co-infection, the presence of one virus affects the description of the other. HIV accelerates the natural course of HBV and HCV infection and promotes the more rapid progression of liver disease to liver disease and hepatocellular carcinoma. In the precast era, the long-term effects of chronic HCV-associated liver disease in co-infected individuals were overshadowed by severe deaths from immunodeficiency-related AIDS. After HAART, indisposition and transience in HIV-infected people were ominously reduced. The magnitudes of liver disease concomitant with chronic HCV infection are of much greater concern today. End-stage disease (ESLD) is currently the leading cause of death in patients with hepatitis virus (HBV)/HIV infection, as well as in patients with co-infection with HCV and HIV. About 400 million people worldwide are chronically infected with the hepatitis B virus. HBV is found in blood and body fluids (semen, saliva, anatomical fluids) and must be transmitted sexually or through contact with infected blood or body fluids. The main route of $\mathrm{HBV}$ transmission in the world is perinatal. Pregnant women are carriers of $\mathrm{HBV}$ and positive for HBeAg hepatitis. In low-endemic areas, the simplest incidence of HBV infection occurs in adolescents and young adults. The most common modes of transmission are STDs and rosacea. All $\mathrm{HCV} / \mathrm{HIV}$ and HBV/HIV patients who are not coinfected with hepatitis A virus (HAV) but are at risk should be vaccinated.

\section{Risk factor of hepatitis $(A, B, C)$ in HIV patient}

HBV is assessed for seven major genotypes, A-G. The seroconversion degrees of hepatitis $E$ virus substance (HBeAg) and therefore disease morbidity and mortality are parallel in patients with genotypes $A$ and D. The chemistry and medical specialties additionally indicate that genotype $A$ patients with $\mathrm{HBeAg}$ seroconversion are more common than their genotype D patients. When acute HBV infection is not inherited in adulthood, $90-95 \%$ of adults display a broad and multispecific cellular response that clears the virus. As a result, it leads to the protection of antibodies to the surface antigen of hepatitis $B$. The mean titer of HAV protein in HIV-positive respondents is approximately 10 times less than in HIV-negative respondents. An absolute lower limit on the level of antibody required to prevent $\mathrm{HAV}$ infection has not been reputable.

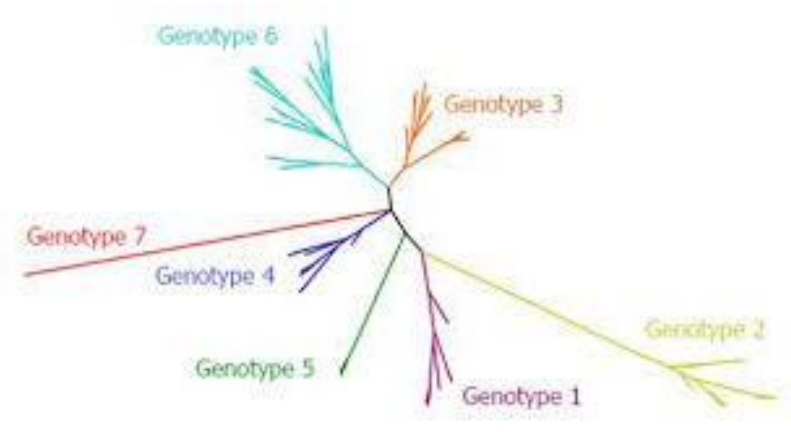

Fig. 1: Genotype of hepatitis C virus (HCV )

Patients not responding to the HAV immunogen should add $\geq 500$ cells $/ \mathrm{mm} 3$ as soon as CD4 counts increase in response to HAART, ideally. The prevalence of antibodies to HCV varies widely among HIV-infected groups, and prevention is difficult because HCV is highly contagious between IDUs. IDU is transmitted in a variety of ways, including partaking needles and syringes, sharing accessories such as stoves, straws, tampons, straps and cotton, sharing medications in regular syringes, and accidental needle sticks. The first method of transmission of HCV is channel and straight up (mother to child). It is rarely sexually pass on. HCV exhibits high global genetic heterogeneity, with six completely different lineages or genotypes distinguished by up to $30 \%$ differences in their genomes. From a medical point of view, infections of genotypes 3 and 4 are further spread between IDUs. HIV-infected patients than single-infected patients. The scattering of genotypes can vary from one region of the planet to another. Since genotypes vary in their sensitivity to quality treatment since of pegylated antiviral agents (PEGIFN) and antiviral agents (RBV) it is important to understand the genotype of each 
patient and therefore the genotype distribution in each country. Why is there no treatment available on the market for all patients with HIV and HCV? Prescribing and treatment guidelines are lacking in many countries, analysis of HCV disease severity is required, treatment requires engineering skills, psychological side effects, and often toxicity. HCV treatment. Treatment is very expensive.

\section{Effect of HIV progression with HAV,HBV, HCV}

HBV-associated HIV has a common route of diffusion in rampant spaces, but $\mathrm{HBV}$ is 100 times additional contagious. Many people with chronic viral hepatitis can develop liver cancer (HCC). Individuals at high risk of developing hepatocellular carcinoma include adult males with cirrhosis of the liver, narrowed by hepatitis B in infancy. HBV contagion in adults usually consists of an premature replicating part with active disease (HBeAg-positive chronic hepatitis $\mathrm{B}$ ), a late low or non-replicating stage with $\mathrm{HBeAg}$ sero convertsion, and diminution or inactivation of liver disease. Because infections share a common route of transmission and HIV accelerates the progression of HBV, HIV testing and referrals should be provided for all HBV patients. Healthcare providers should inform their patients that the description of providing followup has diminished the importance of good clinical care. Conversely, patients have the factual to refuse HIV testing. Patients with decompensated syndrome entail abiding, open-ended management, as setback of the drug after interruption of cure may be one of the swift clinical exacerbations. - ADP is nontoxic in patients with decompensated liver disease and often promotes significant clinical improvement. However, long-term conduct is accompanying with drug confrontation of $28 \%$ after 5 years in patients with a single infection. Therefore, closed HBV polymer monitoring is usually recommended every 6 months to detect drug resistance only in the presence of an infectious agent load. Many studies conclusively confirm that patients with co-infection with HCV and HIV have an additionally faster pathological progression than patients with single infection, even when age, sex, and alcohol use are taken into account. Patients with HCV/HIV co-infection may have a quantitative and/or qualitative deficiency in the immune response to HCV. Spontaneous HCV clearance in HIV-infected patients is significantly lower than in immune proficient patients with acute hepatitis. Because HCV RNA (RNA) may be imperceptible for a brief period during the acute phase of HCV infection, clearance should be confirmed by a sensitive qualitative HCV polymer assay at least twice every 6 months. There are certain contraindications that must be observed even if the immunogen of infectious hepatitis is inactivated and special precautions are not required after vaccination of a person with the disease. - HAV vaccine should not be administered to patients with a history of severe hypersensitivity. Vaccinations in people with moderate to severe acute illness should be delayed until their condition improves. Protection against HAV vaccination during pregnancy and childbirth has not been determined. However, because it is an associate-grade inactivated vaccine, the theoretical risk of traumatic brain injury is low. The risk of vaccination should be compared to the risk of HAV infection. HCV has little or no effect on response to ART or immunological, drug or HIV-related clinical disease. HCV antibodies essentially do not affect progression, but infection with certain genotypes can affect progression. Extensive follow-up in several studies shows that patients taking drug cocktails do not differ significantly in HIV-related mortality from patients with HCV/HIV or HIV alone when given ART . However, there is an increased risk of morbidity and mortality associated with HIV infection by hepatitis B and poor liver health associated with higher hepatotoxicity of ART. Treatment of acute viral hepatitis may reduce the risk of chronic disease. Therefore, if HCV polymers are not cleared from body fluids (confirmed by clinical and/or laboratory studies) at 3 month intervals after disease onset, treatment with pegylated interferon (PEGIFN) for 6 months is recommended. The use of combination therapies in this population remains an area of research. If the co-infected patient has severe immune impairment (CD4 count 200 cells/mm3), the CD4 count should be improved before initiating HCV treatment. If the CD4 count is $200-350$ cells $/ \mathrm{mm} 3$, HCV treatment should be given initially to avoid interactions between the cocktail and HCV drugs and to increase compliance. End of HCV treatment (12 months);

\section{Conclusion}

If an infection is detected, HAART should be initiated. Universal precautions include infection control techniques equivalent to acceptable sterilization of medical and dental equipment, prevention of injection abuse by medical personnel and promotion of safe injections, and strong viral hepatitis immunization recommendations for all patients exposed to co-infectious viruses.

\section{Reference}

1. Kemper CA et al. Safety and immunogenicity of hepatitis A vaccine in human immunodeficiency virusinfected patients: a double-blind, randomized, placebo-controlled trial. Journal of Infectious Diseases, 2003, 187(8):1327-1331.

2. Epidemiology and prevention of vaccine-preventable diseases (the "pink book"), 8th ed. Atlanta, Centers for Disease Control, National Immunization Program, 2004.

3. Hepatitis B. In: Pickering LK, ed. 2003 Report of the Committee on Infectious Disease (the "red book"), 26th ed. Elk Grove Village, IL, American Academy of Pediatrics, 2003:328.

4. Mast EE et al. A comprehensive immunization strategy 
to eliminate transmission of hepatitis B virus infection in the United States. Recommendations of the Advisory Committee on Immunization Practices (ACIP) part 1: immunization of infants, children, and adolescents. Morbidity and Mortality Weekly Report, 2005, 54(RR16):1-31.

5. Mast EE et al. Risk factors for perinatal transmission of hepatitis $\mathrm{C}$ virus (HCV) and the natural history of HCV infection acquired in infancy. Journal of Infectious Diseases, 2005, 192(11):1880-1890.

6. Dore $\mathrm{G}$ et al. Efficacy of tenofovir disoproxil fumarate in antiretroviral therapy-naive and experienced patients coinfected with HIV-1 and hepatitis B virus. Journal of Infectious Diseases, 2004, 189:1185-1192.

7. Nelson M, Portsmouth S, Stebbing J. An open-label study of tenofovir in HIV-1 and hepatitis B virus coinfected individuals. AIDS, 2003, 17:F7-F10.

8. Gish $\mathrm{R}$ et al. Dose range study of pharmacokinetics, safety and preliminary antiviral activity of emtricitabine in adults with hepatitis B virus infection. Antimicrobial Agents and Chemotherapy, 2002, 46:1734-1740. 34. Bang L, Scott L. Emtricitabine. Drugs, 2003, 63:2413-2424.

9. Allavena $\mathrm{C}$ et al. Efficacy and tolerability of a nucleoside reverse transcriptase inhibitor-sparing combination of lopinavir/ritonavir and efavirenz in HIV-1 infected patients. Journal of Acquired Immune Deficiency Syndrome, 2005, 39(3):300-306.

10. Balzarini $\mathrm{J}$ et al. Mechanisms of the potentiating effect of ribavirin on the activity of 2',3'- dideoxyinosine against human immunodeficiency virus. Journal of Biological Chemistry, 1991, 266:21:509-514.

11. Harvie $P$ et al. Ribavirin potentiates the efficacy and toxicity of 2',3'-dideoxyinosine in the murine acquired immunodeficiency syndrome model. Journal of Pharmacology and Experimental Therapeutics, 1996, 279:1009-1017.

12. Japour AJ et al. A phase-1 study of the safety, pharmacokinetics, and antiviral activity of combination of didanosine and ribavirin in patients with HIV-1 disease. Journal of Acquired Immune Deficiency Syndrome, 1996, 13:235-246.

13. Ungo JR et al. Antituberculosis drug-induced hepatotoxicity. The role of hepatitis $C$ virus and the human immunodeficiency virus. American Journal of Respiratory and Critical Care Medicine, 1998, 157(6 Pt 1):1871-1876.

14. Yee $D$ et al. Incidence of serious side-effects from firstline antituberculosis drugs among patients treated for active tuberculosis. American Journal of Respiratory and Critical Care Medicine, 2003, 167(11):1472-1477.

15. Hollingsworth RC et al. Serum HCV RNA levels assessed by quantitative NASBA: stability of viral load over time, and lack of correlation with liver disease. The Trent HCV Study Group. Journal of Hepatology, 1996, 25(3):301-306. 